\title{
Endovascular brachytherapy combined with portal vein stenting and transarterial chemoembolization improves overall survival of hepatocellular carcinoma patients with main portal vein tumor thrombus
}

\author{
Tian-Zhu Yu ${ }^{1,2, *}$, Wen Zhang ${ }^{1,2, *}$, Qing-Xin Liu ${ }^{1,2, *}$, Wen-Hui Li ${ }^{4, *}$, Jing-Qin Ma ${ }^{1,2}$, Zi- \\ Han Zhang ${ }^{1,2}$, Min-Jie Yang ${ }^{1,2}$, Jian-Hua Wang ${ }^{1,2}$, Bing Chen ${ }^{3}$, Shao-Chong Zeng ${ }^{3}$, \\ Jian-Jun Luo ${ }^{1,2}$, Ling-Xiao Liu' ${ }^{1,2}$, Zhi-Ping Yan ${ }^{1,2}$ \\ ${ }^{1}$ Department of Interventional Radiology, Zhongshan Hospital, Fudan University, Shanghai, China \\ ${ }^{2}$ Shanghai Institute of Medical Imaging, Shanghai, China \\ ${ }^{3}$ Department of Radiotherapy, Zhongshan Hospital, Fudan University, Shanghai, China \\ ${ }^{4}$ Department of Interventional Radiology, Yancheng Third People's Hospital, Southeast University, Yancheng, China \\ *These authors have contributed equally to this work \\ Correspondence to: Jian-Jun Luo, email: Luo.jianjun@zs-hospital.sh.cn \\ Ling-Xiao Liu, email: Liu.lingxiao@zs-hospital.sh.cn \\ Zhi-Ping Yan, email: Yan.zhiping@zs-hospital.sh.cn \\ Keywords: hepatocellular carcinoma, main portal vein, tumor thrombus, endovascular brachytherapy, three-dimensional \\ conformal radiotherapy \\ Received: October 20, $2016 \quad$ Accepted: December 27, $2016 \quad$ Published: January 05, 2017
}

\section{ABSTRACT}

Hepatocellular carcinoma (HCC) patients with main portal vein tumor thrombus have a median survival time of only about 4 months. We therefore compared the safety and efficacy of endovascular brachytherapy (EVBT) and sequential threedimensional conformal radiotherapy (3-DCRT). From a cohort of 176 patients, we treated 123 with EVBT using iodine-125 seed strands (group A) and the remaining 53 with sequential 3-DCRT (group B). Overall survival, progression free survival and stent patency characteristics were compared between the two groups. Our analysis demonstrated a median survival of $11.7 \pm 1.2$ months in group A versus $9.5 \pm 1.8$ months in group $B(p=0.002)$. The median progression free survival was $5.3 \pm 0.7$ months in groupA versus $4.4 \pm 0.4$ months in group $B(p=0.010)$. The median stent patency period was $10.3 \pm 1.1$ months in group $A$ versus $8.7 \pm 0.7$ months in group B $(p=0.003)$. Therefore, as compared to sequential 3-DCRT, EVBT combined with portal vein stenting and TACE improved overall survival of HCC patients with main portal vein tumor thrombus.

\section{INTRODUCTION}

Hepatocellular carcinoma (HCC) is the third leading cause of cancer mortality worldwide [1]. Portal venous invasion is frequently encountered with advanced stage HCC [2]. Main portal vein tumor thrombus (MPVTT) increases the metastasis risk, aggravates portal hypertension, and decreases the hepatopedal portal blood flow of the patients [3]. If untreated, the median survival time of these patients is only 2.7 months to 4 months [2].

The occurrence of MPVTT prevents hepatectomy and liver transplantation [4]. Sorafenib demonstrated survival benefits in recent phase III clinical trials and was considered for standard therapy in advanced HCC patients $[5,6]$. However, high costs have limited its use in the developing countries [7]. External beam radiation therapy $[8,9]$ and trans-arterial radioembolization with yttrium-90 microspheres [9] treats $\mathrm{HCC}$ with portal vein thrombosis. However, the blood flow of obstructed MPV could not be restored promptly with either external or internal radiotherapy alone. Trans-arterial chemoembolization (TACE) with or without portal vein stenting can be performed safely in advanced HCC with MPV obstruction $[10,11]$. Nonetheless, the efficacy of TACE for tumor 
Table 1: Baseline characteristics of patients

\begin{tabular}{|c|c|c|c|}
\hline Characteristics & Group A $(N=123)$ & Group B $(N=53)$ & $p$ value \\
\hline Age (years) (SD) & $52.6(10.2)$ & $51.7(9.3)$ & $0.278^{\mathrm{c}}$ \\
\hline Sex (male/female) & $113 / 10$ & $46 / 7$ & $0.296^{\mathrm{d}}$ \\
\hline Etiology of cirrhosis (HBV/HCV/alcoholic/cholestasis) & $98 / 14 / 9 / 2$ & $35 / 11 / 6 / 1$ & $0.267^{\mathrm{d}}$ \\
\hline HCC morphology (multifocal/diffuse) & $98 / 25$ & $38 / 15$ & $0.247^{\mathrm{d}}$ \\
\hline $\mathrm{HCC}$ maximum diameter $(\mathrm{cm})(\geq 5 /<5)$ & $79 / 44$ & $34 / 19$ & $0.992^{\mathrm{d}}$ \\
\hline Location of TT(LIPV + MPV/RIPV + MPV) & $42 / 81$ & $15 / 38$ & $0.447^{\mathrm{d}}$ \\
\hline Degree of MPVTT ${ }^{\text {a }}$ (stenosis/occlusive) & $93 / 30$ & $40 / 13$ & $0.984^{\mathrm{d}}$ \\
\hline $\operatorname{AFP}(\mathrm{ng} / \mathrm{ml})(>400 / \leq 400)$ & $80 / 43$ & $33 / 20$ & $0.724^{\mathrm{d}}$ \\
\hline Child-Pugh grade (A/B) & $111 / 12$ & $45 / 8$ & $0.306^{\mathrm{d}}$ \\
\hline ECOG PS $(0 / 1 / 2)$ & $10 / 82 / 31$ & $4 / 31 / 18$ & $0.491^{\mathrm{d}}$ \\
\hline $\begin{array}{l}\text { Previous treatment (No/Resection/TACE/RFA/Combination } \\
\text { therapy }^{\mathrm{b}} \text { ) }\end{array}$ & $81 / 12 / 16 / 6 / 8$ & $30 / 4 / 10 / 7 / 2$ & $0.237^{\mathrm{d}}$ \\
\hline
\end{tabular}

Abbreviations: AFP Alpha-fetoprotein, HBV hepatitis B virus, HCC hepatocellular carcinoma, HCV hepatitis C virus, LIPV left intrahepatic portal vein, MPV main portal vein, MPVTT main portal vein tumor thrombus, RFA radiofrequency ablation, RIPV right intrahepatic portal vein, SD standard deviation, TACE transarterial chembolization, TT tumor thrombus.

a If the diameter of the filling defect exceeded $90 \%$ of MPV's on the transverse section image of contrast-enhanced CT or MRI before therapy, this patient's MPV was arbitrarily defined as occlusive.

${ }^{\mathrm{b}}$ Combination therapy means surgical resection followed by TACE or RFA or both and TACE combined with RFA

${ }^{\mathrm{c}}$ Independent $t$ test was used

${ }^{\mathrm{d}}$ Chi-square test was used

thrombus, which often lacks tumor feeding arteries, is controversial $[5,6,12]$. Thus, novel effective treatment modalities need to be explored.

Recently, endovascular brachytherapy (EVBT) with Iodine-125 seed strand implantation was reported to be safe for advanced HCC with main portal vein tumor thrombosis [13-15]. Meanwhile, the combination of sequential three-dimensional radiotherapy (3-DCRT) with portal vein stenting and TACE was also reported to beneficial for these patients [16]. Since these two treatment regimens have not been compared, we analyzed the safety and efficacy of portal vein stenting and TACE combined with EVBT or sequential 3-DCRT to treat HCC with MPVTT in this study.

\section{RESULTS}

\section{Patient data}

Most patients included in this study were males with a mean age of $52.4 \pm 9.9$ years (range $28-75$ years). Cirrhosis, secondary to hepatitis B was recorded for over $79 \%$ of the patients. We also recorded multifocal HCC in $136(77.3 \%)$ patients and diffuse HCC in $40(22.7 \%)$ patients. More than $60 \%$ patients demonstrated maximal diameter of HCC greater than $5 \mathrm{~cm}$. Tumor thrombus that extended from the right intrahepatic portal vein branches into MPV was more frequently encountered than the left. Stenosis and occlusion of the MPV was found in $133(75.6 \%)$ and $43(24.4 \%)$ patients, respectively. The baseline characteristics of patients showed no significant difference between the two groups (Table 1).

\section{Portal vein stenting, EVBT, SPECT/CT scan and sequential 3-DCRT data}

The mean length of the obstructed MPV was 55.4 $\pm 25.8 \mathrm{~mm}$ (range 10-170 $\mathrm{mm}$ ) in Group A and $56.6 \pm$ $23.6 \mathrm{~mm}$ (range $10-100 \mathrm{~mm})$ in Group B $(P=0.747)$. After stent placement, the mean pressure of the MPV dropped from $40.6 \pm 5.2 \mathrm{~cm} \mathrm{H}_{2} \mathrm{O}$ (range $28-55 \mathrm{~cm} \mathrm{H}_{2} \mathrm{O}$ ) to $34.5 \pm 5.0 \mathrm{~cm} \mathrm{H}_{2} \mathrm{O}$ (range $\left.25-44 \mathrm{~cm} \mathrm{H}_{2} \mathrm{O}\right)(P<0.001)$ in group $\mathrm{A}$ and from $41.8 \pm 5.8 \mathrm{~cm} \mathrm{H}_{2} \mathrm{O}$ (range $31-57 \mathrm{~cm}$ $\left.\mathrm{H}_{2} \mathrm{O}\right)$ to $35.3 \pm 4.9 \mathrm{~cm} \mathrm{H}_{2} \mathrm{O}$ (range $\left.26-45 \mathrm{~cm} \mathrm{H}_{2} \mathrm{O}\right)(P<$ 0.001 ) in group B. A mean number of $16.1 \pm 5.3$ (range 6-26) Iodine-125 seeds were implanted in the MPV of group A patients. A mean $162.3 \pm 21.8$ Gy (range 81.6 $-192.0 \mathrm{~Gy}$ ) dose of radiation was prescribed to the tumor thrombus in group A based on the formula provided by the American Association Physicists in Medicine [17] and the Iodine-125 Radiation Field Distribution Calculation software (version 0.11, Shanghai Medical Radiation 
Research Institute) used by Zhang [18] and Chen [19]. The $\mathrm{SPECT} / \mathrm{CT}$ scans showed that all stents and radioactive Iodine-125 seeds strands had been placed in the obstructed MPV correctly without displacement in the group A patients. Within 2-6 weeks, a mean radiation dose of 51.4 \pm 8.4 Gy (range $20-66$ Gy) was delivered by 3 -DCRT to the group B patients.

\section{Tumor response to TACE procedures}

A mean number of $3.3 \pm 1.9$ sessions of TACE (range 1-9) were performed in Group A and $3.6 \pm 2.2$ (range $1-10)$ in Group B $(P=0.231)$ patients. The mean dose of epirubicin and iodized oil used in the TACE procedure was $26.7 \pm 7.1 \mathrm{mg}$ (range $10-40 \mathrm{mg}$ ), 9.5 $4.1 \mathrm{ml}$ (range 2-20 ml) in Group A and $26.0 \pm 7.9 \mathrm{mg}$ (range 10-40 mg), $9.3 \pm 4.3$ (range $3-16 \mathrm{ml}$ ) in Group B $(P=0.557$ and 0.771$)$, respectively. The objective HCC response rate $(\mathrm{CR}+\mathrm{PR})$ was $19.5 \%$ in group $\mathrm{A}$ and 17.0 $\%$ in group $\mathrm{B}(P=0.693)$.

\section{Treatment-related complications}

No complications related to stent deployment and Iodine-125 seeds strand implantation, such as intraperitoneal bleeding, stent displacement and radioactive seeds dislodgement, were recorded. Postchemoembolization syndrome, including fever, vomiting and right upper abdominal pain, was observed in almost all patients. No statistical difference was found between the two groups. All the symptoms resolved within $3-5$ days after symptomatic treatments. A transient increase of aminotransferase and bilirubin after the procedures was recorded. No grade 3 or 4 radiation-induced toxicity occurred.

\section{Overall survival analysis}

During a mean follow-up time of $11.7 \pm 8.3$ months (range 1.2 - 32.0 months), 95 (77.2\%) and $48(90.6 \%)$ patients died in group $\mathrm{A}$ and $\mathrm{B}$, respectively $(P=0.038)$. The mean and median survival times were $15.1 \pm 1.0$ months (95\% CI 13.2 - 17.1 months) and $11.7 \pm 1.2$ months (95\% CI 9.3-14.1 months) in group A compared to $10.4 \pm 1.0$ months (95\% CI $8.5-12.2$ months) and 9.5 \pm 1.8 months (95\% CI $5.9-13.1$ months) in group B. The 12 - and 24-month cumulative survival rates were $48.7 \%$ and $26.1 \%$ in group A and $31.4 \%$ and $3.4 \%$ in group B, respectively $(P=0.002)$ (Figure $1 \mathrm{~A})$. EVBT, stent patency and variceal bleeding were identified as independent

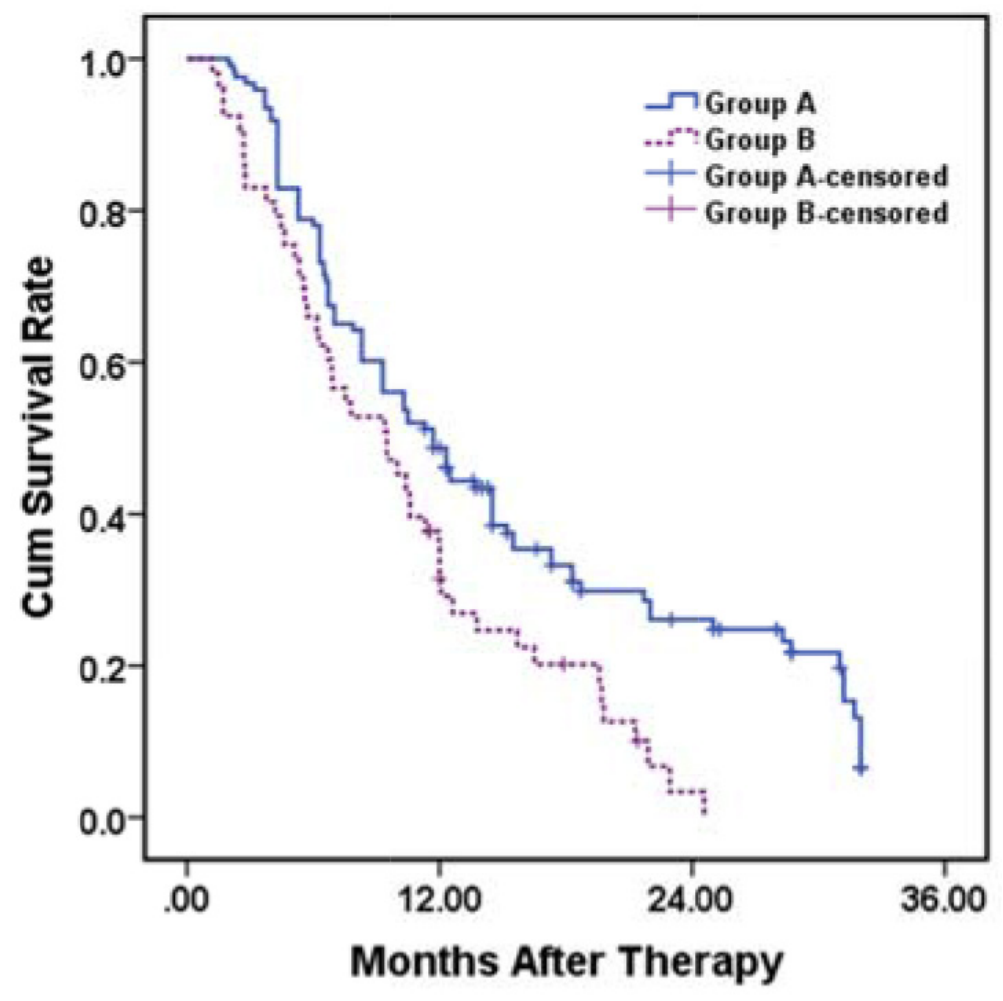

Figure 1A: Kaplan-Meier analysis for overall survival in group A (with EVBT) versus group B (with 3-DCRT). Mean and median survival time were $15.1 \pm 1.0$ months (95\% CI 13.2 - 17.1 months) and $11.7 \pm 1.2$ months (95 \% CI 9.3-14.1 months) in group A compared to $10.4 \pm 1.0$ months (95\% CI $8.5-12.2$ months) and $9.5 \pm 1.8$ months ( $95 \%$ CI $5.9-13.1$ months) in group B, respectively. The 12- and 24-month cumulative survival rates were $48.7 \%, 26.1 \%$ in group A and $31.4 \%, 3.4 \%$ in group B, respectively $(p=0.002, \log$ rank test). 


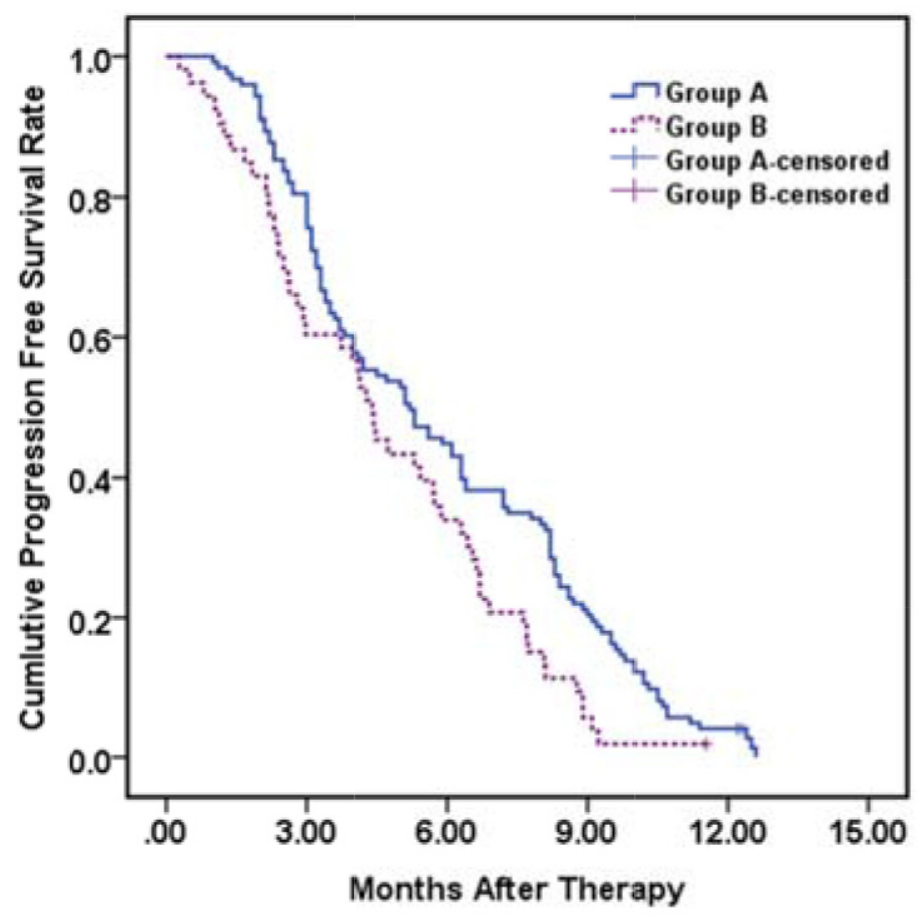

Figure 1B: Kaplan-Meier analysis for progression free survival in group A (with EVBT) versus group B (with 3-DCRT). Mean and median progression free survival times were $5.8 \pm 0.3$ months (95\% CI $5.3-6.4$ months) and $5.3 \pm 0.7$ months (95\% CI 3.8-6.6 months) in group A compared to $4.7 \pm 0.4$ months (95 \% CI 4.0 - 5.4 months) and $4.4 \pm 0.4$ months (95\% CI $3.6-5.2$ months) in group B $(p=0.010, \log$ rank test $)$.

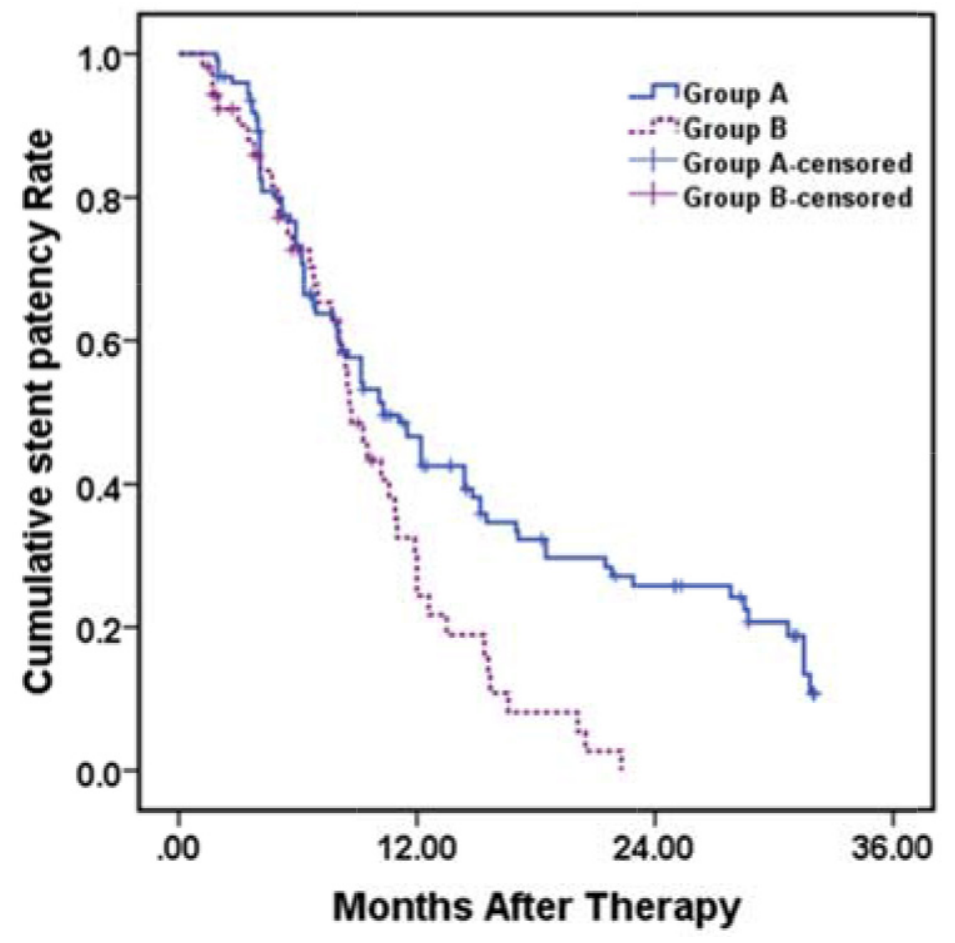

Figure 1C: Kaplan-Meier analysis for stent patency period in group A (with EVBT) versus group B (with 3-DCRT). Mean and median stent patency period were $14.7 \pm 1.0$ months (95\% CI 12.7-16.8 months), $10.3 \pm 1.1$ months (95\% CI $8.1-12.5$ months) in group A and $9.6 \pm 0.8$ months (95\%CI 8.1-11.2 months), $8.7 \pm 0.7$ months (95\% CI $7.4-10.0$ months) in group B, respectively. The 12- and 24-month cumulative stent patency rates were $46.5 \%, 25.7 \%$ in group A and $29.8 \%, 0 \%$ in group $\mathrm{B}$, respectively $(p=0.003, \log$ rank test). 
Table 2: Predictors for survival in univariate and multivariate analysis

\begin{tabular}{|c|c|c|c|c|c|c|}
\hline \multirow{2}{*}{ Variable } & \multicolumn{2}{|c|}{ Univariate Analysis } & \multicolumn{4}{|c|}{ Multivariate Analysis } \\
\hline & HR & $95 \% \mathrm{CI}$ & p-Value & HR & $95 \%$ CI & $p$-Value \\
\hline Age (years) $(\geq 55 /<55)$ & 1.080 & $0.775-1.506$ & 0.649 & & & \\
\hline Sex (male/female) & 1.042 & $0.576-1.884$ & 0.893 & & & \\
\hline $\begin{array}{l}\text { HCC morphology (multifocal/ } \\
\text { diffuse) }\end{array}$ & 0.735 & $0.488-1.107$ & 0.140 & & & \\
\hline $\begin{array}{l}\text { Etiology of cirrhosis (With/Without } \\
\text { hepatitis) }\end{array}$ & 0.955 & $0.755-1.209$ & 0703 & & & \\
\hline $\begin{array}{l}\text { HCC maximum diameter }(\mathrm{cm}) \\
(>=5 /<5)\end{array}$ & 1.054 & $0.743-1.496$ & 0.768 & & & \\
\hline $\begin{array}{l}\text { Location of TT(LIPV + MPV/ } \\
\text { RIPV + MPV) }\end{array}$ & 0.958 & $0.673-1.363$ & 0.811 & & & \\
\hline $\begin{array}{l}\text { Degree of MPVTT (stenosis/ } \\
\text { occlusive) })^{\mathrm{a}}\end{array}$ & 0.876 & $0.597-1.286$ & 0.500 & & & \\
\hline $\operatorname{AFP}(\mathrm{ng} / \mathrm{ml})(>400 / \leq 400)$ & 1.328 & $0.934-1.889$ & 0.114 & & & \\
\hline Child-Pugh grade (A/B) & 0.824 & $0.495-1.371$ & 0.455 & & & \\
\hline ECOG PS $(0$ and $1 / 2)$ & 1.098 & $0.827-1.457$ & 0.518 & & & \\
\hline Previous treatment (Yes/No) & 1.080 & $0.922-1.264$ & 0.341 & & & \\
\hline Therapy (EVBT/3D-CRT) & 0.581 & $0.407-0.830$ & $0.003 *$ & 0.649 & $0.448-0.938$ & $0.022 *$ \\
\hline Stent (Patent /Occlusive) & 0.477 & $0.316-0.718$ & $<0.001 *$ & 0.553 & $0.360-0.849$ & $0.007 *$ \\
\hline $\mathrm{HCC}$ response $(\mathrm{CR}+\mathrm{PR} / \mathrm{SD}+\mathrm{PD})$ & 0.994 & $0.649-1.522$ & 0.979 & & & \\
\hline $\begin{array}{l}\text { Variceal bleeding after therapy } \\
\text { (Yes/No) }\end{array}$ & 1.930 & $1.338-2.784$ & $<0.001 *$ & 1.907 & $1.316-2.763$ & $0.001 *$ \\
\hline $\begin{array}{l}\text { Liver function decompensation } \\
\text { after therapy (Yes/No) }\end{array}$ & 1.104 & $0.721-1.690$ & 0.648 & & & \\
\hline
\end{tabular}

Abbreviations: AFP Alpha-fetoprotein, CI confidence interval, CR complete response, ECOG PS Eastern Cooperative Oncology Group Performance Status, EVBT endovascular brachytherapy, LIPV left intrahepatic portal vein, HCC hepatocellular carcinoma, HR hazard ratio, MPV main portal vein, MPVTT main portal vein tumor thrombus, PD progressive disease, PR partial response, RIPV right intrahepatic portal vein, SD stable disease, TT tumor thrombus * A $p$ value $<0.05$ indicated a significant difference

${ }^{\text {a }}$ Diameter of the filling defect exceeded $90 \%$ of MPVs on the transverse section image of contrast-enhanced CT or MRI before therapy was defined as occlusive

predictors of patient's survival in both the univariate and multivariate analysis (Table 2).

\section{Progression free survival analysis}

During the course of the study, occurrence of either intra-hepatic/extra-hepatic HCC spread, variceal bleeding, liver function decompensation or occurrence of more than one of these events were observed in 59 (48.0\%), 30(24.4\%), $11(8.9 \%)$ and $12(9.5 \%)$ in group A and 21(39.6\%), $16(30.2 \%), 7$ (13.2\%) and 4 (7.5\%) patients in group $\mathrm{B}$, respectively $(P=0.752)$. The mean and median progression free survival time were $5.8 \pm 0.3$ months (95\% CI $5.3-6.4$ months) and $5.3 \pm 0.7$ months
(95\% CI 3.8-6.6 months) in group A compared to 4.7 \pm 0.4 months $(95 \%$ CI $4.0-5.4$ months $)$ and $4.4 \pm 0.4$ months ( $95 \%$ CI $3.6-5.2$ months) in group B $(P=0.010)$ (Figure 1B).

\section{Stent patency data}

During the follow-up, stent occlusion was observed in $87(70.7 \%)$ patients in group A and 41 (77.4\%) patients in group $\mathrm{B}(P=0.365)$. The mean and median stent patency periods were $14.7 \pm 1.0$ months $(95 \%$ CI 12.7 16.8 months) and $10.3 \pm 1.1$ months (95\% CI 8.1-12.5 months) in group A and $9.6 \pm 0.8$ months $(95 \%$ CI $8.1-$ 11.2 months) and $8.7 \pm 0.7$ months (95\% CI $7.4-10.0$ 


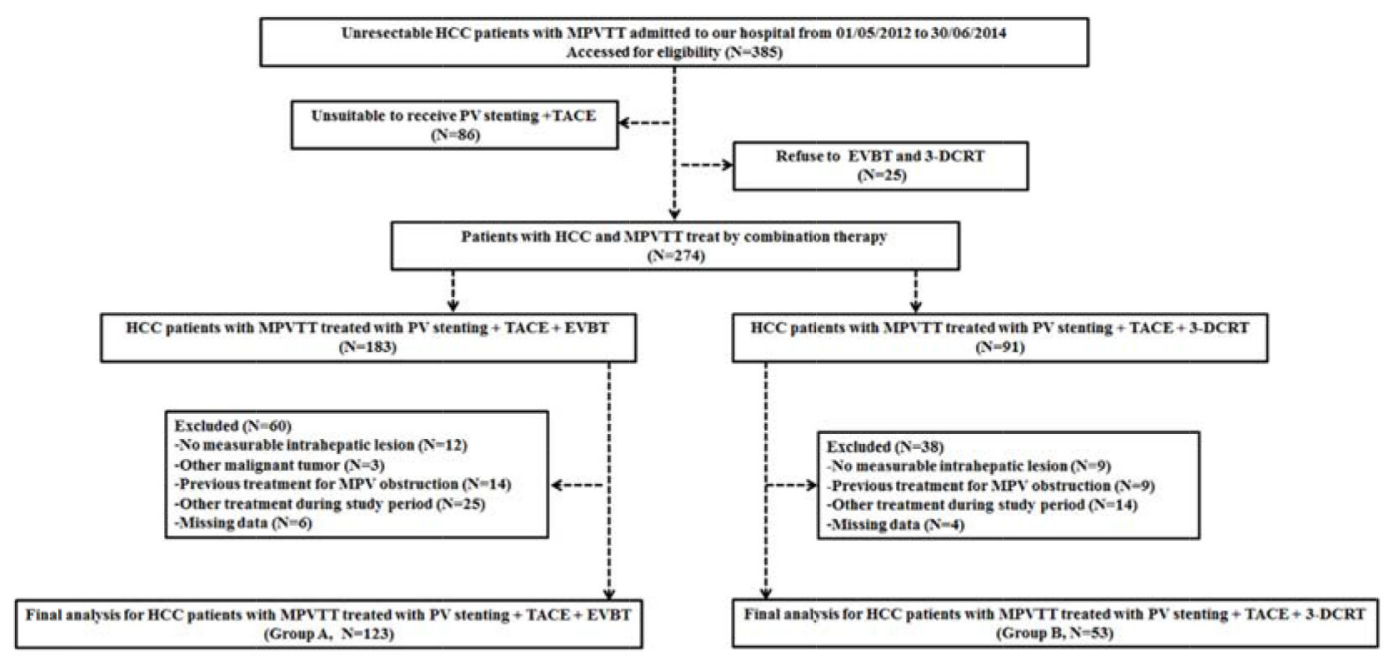

Figure 2: The flow diagram of patients enrolled in the study. EVBT = Endovascular brachytherapy, 3-DCRT = Three-dimensional conformal radiotherapy, HCC $=$ Hepatocellular carcinoma, MPV $=$ Main portal vein, MPVTT $=$ Main portal vein tumor thrombus, $\mathrm{PV}=$ Portal vein, $\mathrm{SMV}=$ Superior mesenteric vein, $\mathrm{SV}=$ Splenic vein, $\mathrm{TACE}=$ Transarterial chemoembolization, $\mathrm{TT}=\mathrm{Tumor}$ thrombus.
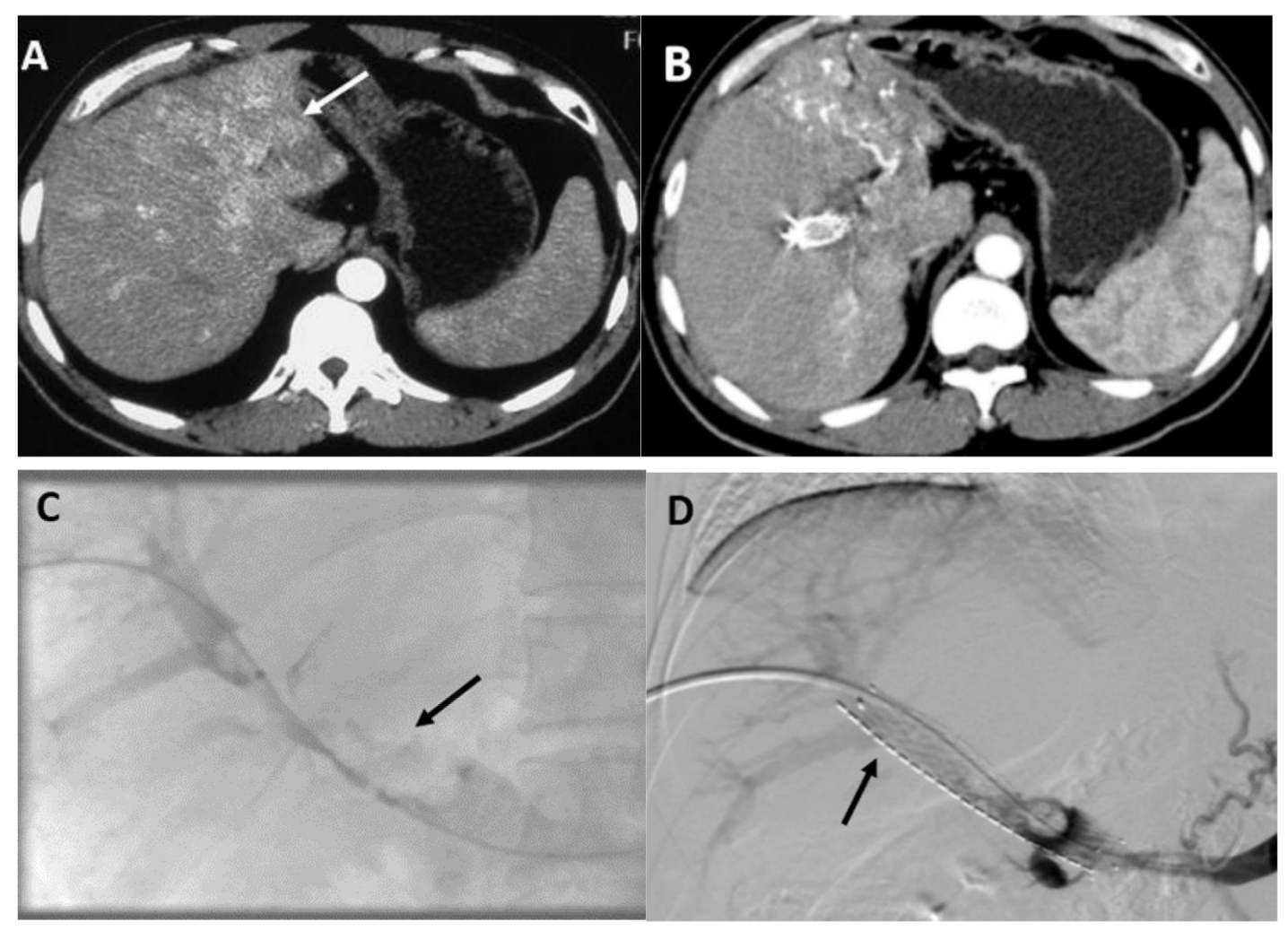

Figure 3: Images of portal vein stenting and TACE combined with endovascular brachytherapy performed in a 39-year-old male patient (group A). A. An invasive HCC (white arrow) detected on the left lobe by the enhanced abdominal CT scan before therapy. B. Image of an enhanced abdominal CT scan performed one month after the first therapy. Atrophic left lobe of liver and partial response of HCC to TACE was observed. C. The tumor thrombus (black arrow) in his MPV was observed on the direct portography after his right patent intrahepatic portal vein branch was punctured. D. Image captured after a 14/100 mm stent and iodine-125 seed strand that was loaded with 20 radioactive seeds (black arrow) had been implanted in his MPV showing restoration of the flow of the obstructed MPV. 
months) in group B, respectively. The 12- and 24-month cumulative stent patency rates were $46.5 \%$ and $25.7 \%$ in group $\mathrm{A}$ and $29.8 \%$ and $0 \%$ in group $\mathrm{B}$, respectively $(P=$ 0.003 ) (Figure 1C).

\section{DISCUSSION}

Although the survival of patients with $\mathrm{HCC}$ has improved during recent decades [1], the prognosis of $\mathrm{HCC}$ that is complicated by MPVTT remains extremely poor [4]. The optimal treatment modality for this group of HCC patients has not yet been established [2].

Sorafenib was recommended for advanced HCC $[5,6]$ and TACE was reported to benefit patients with HCC and portal vein tumor thrombosis [10]. However, the patients treated with TACE alone [20], sorafenib monotherapy [21] and TACE combined with sorafenib [22-24] demonstrated poor overall survival times of 4.1, 3.0 and 3.0-7.0 months, respectively. To further the survival benefit for these patients, restoration of the hepato-petal portal vein blood flow and reduction of tumor thrombus burden were postulated. Portal vein stenting was used widely to treat MPV obstruction caused by both benign and malignant diseases $[11,16]$. However, in-stent tumor growth and neointimal hyperplasia caused stent failure. Radiation therapy inhibited tumor cell proliferation and induce apoptosis [13, 25]. Especially, continuous low dose rate radiotherapy had anti-neointimal hyperplasia effect that could prolong the patency period of the stent [26]. Moreover, low dose rate of radiation had been reported to decrease the incidence of metastasis by altering the immunophenotype of the tumor cells [27, 28]. Most importantly, EVBT and sequential 3-DCRT were capable of inhibiting MPVTT [14-16, 29]. However,
MPVTT treated by these two different types of radiation had never been directly compared.

In our cohort, Iodine-125 seeds strands were successfully placed in the obstructed MPV of group A patients. The maximal tolerance radiation dose of the vessels was reported to be more than 800 Gy [30]. In our study, a mean of $162.3 \pm 21.8$ Gy radiation dose was tolerated by all group A patients. Although this dose was higher than that in group B patients, no grade 3 or 4 radiation-induced toxicity was observed during the followup. This was probably because the radiation emitted by Iodine-125 seed was at a low dose rate and short tissuepenetrating distance.

Compared with external radiotherapy, brachytherapy with Iodine-125 seed implantation had the following advantages: (i) It had highly accumulated radiation within the tumor area without serious damage to the surrounding normal tissue; (ii) Sustained radiation inhibited tumor cell proliferation and induced apoptosis; (iii) It was not affected by the patient's respiration motion; (iv) A low dose rate radiation decreased the incidence of metastasis by altering the immunophenotype of tumor cells [28] and (v) It was convenient to patients as it was a one-stop treatment method.

Our data showed that the overall survival extended significantly in group A patients. Multivariate analysis showed that patients that were treated with EVBT with patent stents and no variceal bleeding had better survival (Table 2). The longer survival time in group A patients can be attributed to the anti-tumor and anti-neointimal hyperplasia effects of sustained low dose rate radiation emitted by the Iodine-125 seeds strands [26]. These prolonged the patency period of the stent and decreased the risk of second variceal bleeding.
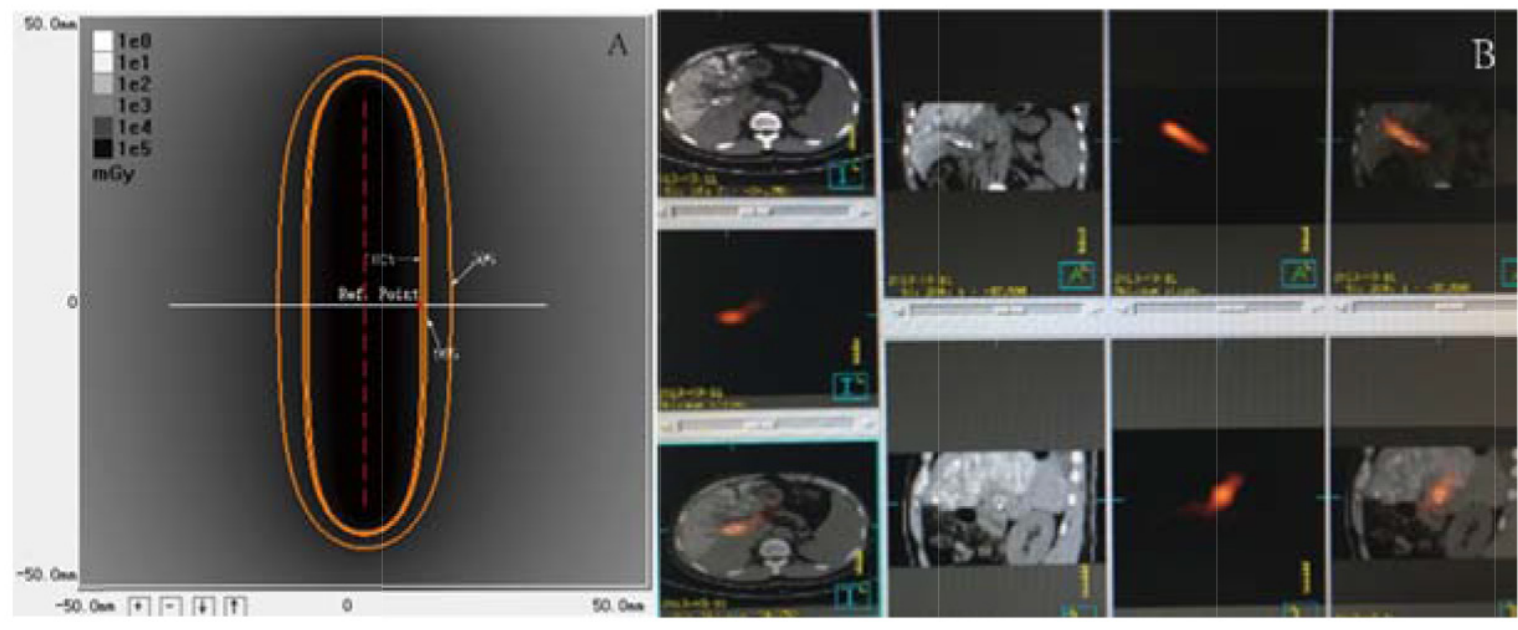

Figure 4: Images of dosimetry of a Iodine-125 seeds strand and SPECT/CT. A. Dosimetry of a seed strand containing 16 Iodine-125 seeds. The isodose contours are: 100\% (62.5Gy, reference point, red dot), $90 \%$ (56.2Gy), and $50 \%$ ( $31.2 \mathrm{~Gy})$. The 240 day accumulation dose was141.6Gy. B. Image of a SPECT/CT scan performed 1 day after the procedure. Stent and I-125 seed strands were implanted correctly in the MPV without displacement. Radiation emitted by a I-125 seed strand was distributed homogeneously and completely covered the target lesion. 
Direct MPV puncturing for radioactive seed implantation was reported to treat MVPTT [31]. Iodine-125 seeds were aligned linearly and sealed into a 4-F catheter continuously to construct a seeds strand in our study to prevent seed dislodgment and ensure that the target lesion was covered by the radiation. Furthermore, single portal vein puncture site reduced bleeding complications. In fact, there were no bleeding complications recorded in Group A. We observed that the hepato-pedal portal flow was restored and the portal vein pressure decreased immediately after stenting. This increased the safety of subsequent TACE in patients with compromised portal blood supply [12].

Since this was a single-center retrospective study, potential selection bias cannot be excluded. Therefore, a well-designed prospective randomized trial with sufficient sample size is necessary to confirm the efficacy and superiority of portal vein stenting and TACE combined with EVBT for the treatment of patients with $\mathrm{HCC}$ and MPV tumor thrombus. Recently, Matsuo Y and colleagues reported that SBRT with hypofractionated technique might be superior to conventional three-dimensional conformal radiotherapy for the treatment of PVTT [35]. However, the SBRT technique was not available for our research study and hence in future SBRT and brachytherapy need to be comparatively investigated.

In conclusion, this study showed that portal vein stenting and TACE combined with EVBT could be performed safely in patients with HCC and MPVTT. Compared to sequential 3-DCRT, Iodine-125 seeds strands implantation significantly prolonged stent patency, extended the progression free survival time and improved the overall survival of patients with HCC and MPVTT.

\section{MATERIALS AND METHODS}

\section{Study design and patient selection}

This study was approved by the ethics committee and institutional review board (2009-080) of our institution. All procedures were followed in accordance with the ethical standards of the responsible committee on human experimentation (Zhongshan Hospital, Fudan University, China) and the Helsinki Declaration of 1975, as revised in 2008 (5). Informed consent was obtained from all patients included in the study. 385 patients diagnosed with HCC and MPVTT between May 2012 and June 2014 were referred to our institution. Among them, 86 patients were judged unsuitable to receive PV stenting and TACE (patients with ChildPugh classification $\mathrm{C}$ or with Eastern Cooperative Oncology Group performance status of 3 or 4) and 25 patients refused EVBT or 3-DCRT. Therefore, 183 patients underwent EVBT combined with portal vein stenting and TACE and 91 patients received sequential 3-DCRT combined with portal vein stenting and TACE.
Patients with no measurable intrahepatic lesions (12 in EVBT group and 9 in 3-DCRT group), other concurrent malignancies (3 in EVBT group), previous MPVTT treatment history (14 in EVBT group and 9 in 3-DCRT group), other treatments after the procedure (25 in EVBT group and 14 in 3-DCRT group) and missing followup clinical data (6 in EVBT group and 4 in 3-DCRT group) were excluded. Ultimately, 123 patients treated with EVBT (Group A) and 53 with 3-DCRT (Group B) were included in the final data analysis (Figure 2). Other eligible criteria were as follows: (i) age >18 years; (ii) $\mathrm{HCC}$ was diagnosed according to the European Association for the Study of Liver/American Association for the study of Liver Disease guidelines [32]; (iii) tumor thrombus, a low-attenuation intraluminal filling defect extending from intrahepatic portal vein branches adjacent to the primary tumor into MPV was confirmed by contrast-enhanced abdominal computer tomography (CT) or magnetic resonance imaging (MRI) (Figure 3A); (iv) patency of one or two of the second-order intrahepatic portal vein branch (v) Child-Pugh classification grade A or B; (vi) Eastern Cooperative Oncology Group (ECOG) performance status less than or equal to 2 and (vii) No contraindication for TACE, such as HCC burden $>70$ $\%$ of total liver volume, high-flow intrahepatic arterial venous shunt and/or serious coagulant disorder. Patients were excluded from this study if (i) no measurable intrahepatic lesion could be observed; (ii) they had concurrent malignancy other than HCC; (iii) previous therapy was performed for MPV obstruction (such as stent placement, radiotherapy or sorafenib); (iv) they received other treatment (radiofrequency ablation or molecular targeted drug therapy) besides the above mentioned therapy during the course of this study or (v) the data of follow-up were missing. Either EVBT or radiation was considered to be beneficial for patients with $\mathrm{HCC}$ and tumor thrombus in the main portal vein but not as a standard therapeutic option. According to the Chinese expert consensus on multidisciplinary diagnosis and treatment of hepatocellular carcinoma with portal vein tumor thrombus, the treatment choices for patients with MPVTT include surgery, radiotherapy and/or TACE depending on the patient's preference [33]. Before the procedure, the benefits and potential adverse events related to endovascular brachytherapy (EVBT) and radiation were explained in detail to the patients and informed consents were signed. Therefore, the decision to receive EVBT or radiation was entirely based on the patient's own will.

\section{Stent and Iodine-125 seed characteristics}

Self expanding Nitinol stents (Luminxx III, Bard, Covington, GA) with a diameter of $12-14-\mathrm{mm}$ and length of $60-100 \mathrm{~mm}$ were used in this study. The model 6711 Iodine-125 seeds (XinKe, Shanghai, China) used 
in this study were a cylindrical brachytherapy source with an active length of $3.25 \mathrm{~mm}$ encapsulated by titanium. The diameter and the length of the titanium capsule were $0.8 \mathrm{~mm}$ and $4.5 \pm 0.5 \mathrm{~mm}$, respectively. The radioactivity of each Iodine-125 seed was 25.9 $\mathrm{MBq}$ with a half-life of 59.4 days. The principal photon emissions were 27.4-, 31.4-keV X-ray and $35.5-\mathrm{keV}$ gamma-ray. The half-value thickness of tissue for Iodine-125 seeds was $17 \mathrm{~mm}$ and the incipient dose rate was $7 \mathrm{cGy} / \mathrm{h}$. Seeds were arranged linearly and sealed into a 4-F catheter continuously to construct an Iodine-125 seeds strand. The dose reference point was chosen at $10 \mathrm{~mm}$ from the axis of the iodine- 125 seed strand source $(Z=0, r=10 \mathrm{~mm})$. The 240 days accumulating dose of the reference point was calculated by an iodine-125 Radiation Field Distribution Calculation software (version 0.1, Institute of Radiation Medicine, Fudan University, Shanghai, China) based on the American Association of Physicists in Medicine TG43U1 brachytherapy formula (Figure 4A).

\section{Portal vein stenting and Iodine-125 seeds strand placement procedures}

After local anesthesia, a tumor-free second-order branch of the intrahepatic portal vein was punctured using a 22-gauge Chiba needle (Cook, Inc, Bloomington, Indiana) guided by ultrasound. A 6-F NEFF set (Cook) was introduced into the intrahepatic portal vein over a 0.018-inch wire (Cook). Then, a 0.035-inch, 150-cmlong wire (Terumo, Tokyo, Japan) combined with a 4-F Cobra catheter (Cordis, Miami Lakes, FL) was manipulated to cross the stenotic segment to the superior mesenteric vein (SMV) through the outer cannula of the NEFF set. The Cobra catheter was removed and the outer cannula of the NEFF set was replaced by a 7-F, 23-cm-long sheath (Cordis) over the wire. Portography was performed to measure the diameter and length of the stenotic segment by a 5-F calibrated pigtail catheter (Cook) placed in the splenic vein (Figure $3 C)$. The portal vein pressure was measured at the distal and the proximal end of the obstructed MPV. If gastroesophageal varices were opacified, the left gastric vein or/and short gastric vein were embolized with coils (Cook). The number of Iodine-125 seeds that needed to be implanted was calculated by the following formula: $\mathrm{N}=$ length of obstructed MPV $(\mathrm{mm}) / 4.5+4$. After intravenous administration of $50 \mathrm{U} / \mathrm{kg}$ heparin (XingYi, Shanghai, China), two 0.035-inch, 260-cm-long stiff wires (Terumo) were inserted into SMV through the 7-F sheath. After the sheath had been removed, the outer cannula of the NEFF set and a self-expandable stent was introduced to the MPV over one of the stiff wires. The stent was deployed from distal MPV into the patent intrahepatic portal vein. Through the outer cannula of the NEFF set, the Iodine-125 seeds strand was delivered to the target position and released between the stent and the MPV. Portography and pressure measurements were performed again (Figure 3D). Finally, the transhepatic puncture track was occluded by coils (Cook).

\section{TACE methodology}

To identify all the feeding arteries of the tumor, angiography of the celiac, hepatic, superior mesenteric, left gastric and bilateral inferior phrenic artery was performed by a 5-F RH catheter (Cook). The target artery was catheterized with a 2.7-F microcatheter (Renegade, Boston Scientific, Natick, MA). $10-50 \mathrm{mg} / \mathrm{m}^{2}$ of epirubicin (Pharmorubicin, Pfizer, New York, NY) was mixed with 5-20 ml of iodized oil (Lipiodol Ultrafluide, Laboratoire Guerbet, Aul-nay-sous-Bois, France) by using a pumping method. The dose of epirubicin and iodized oil was determined based on the patient's liver function and tumor vascularity. Under fluoroscopic monitoring, the mixture was infused at a rate of $0.5-1$ $\mathrm{ml} / \mathrm{min}$ through the microcatheter until stasis flow in the tumor vascularity was achieved. Finally, gelatin sponge (Jingling, Jiangsu, China) was used to embolize the feeding artery.

\section{Post-procedure management strategy}

After the procedure, tropisetron hydrocloride (Novartis Pharma Schweiz AG, $5 \mathrm{mg}$ /day), omeprazole (Changzhou Pharmacectic, Jiangsu, China $40 \mathrm{mg} /$ day) and ornithine aspartate (Merz Pharma, GmbH \& Co. $\mathrm{KGaA}$, Frankfurt, Germany, $10 \mathrm{~g}$ /day) were administered intravenously to all patients for 3-5 days. Pain and fever attributed to post-embolization syndrome were controlled individually using non-steroidal anti-inflammatory drugs or opioids. 5,000 U of low molecular-weight heparin (XinYi, Shanghai, China) was injected subcutaneously twice a day. One day after the procedure, single-photon emission computer tomography (SPECT) combined with a CT (SPECT/CT) scan was performed to evaluate the distribution of radiation emitted by the Iodine-125 seeds strand implanted in the MPV (Figure 4B). Warfarin (XinYi, Shanghai, China), starting with $2.5 \mathrm{mg}$ every day, was prescribed to all the patients, 3 days after the procedure for 6 months and the dosage was adjusted based on the coagulation function test (international normalized ratio $=1.8-2.0)$ performed weekly.

\section{Sequential 3-DCRT methodology}

The method of stent implantation combined with TACE and the protocol of post-procedure management in group B was the same as in group A. Sequential 3-DCRT was performed two weeks after the above mentioned therapy. The CT data of the patients was transferred to a 3-DCRT treatment planning system (Pinnacle 7.6C). The gross target volume (GTV) included the stent. The CTV 
was defined as the volume of stent placed in the MPV (GTV) plus a small margin of 3-5 $\mathrm{mm}$. Intra-hepatic tumor was not included in the CTV. The planning target volume (PTV) was CTV plus 5-10 mm margin to account for daily setup error and target motion. A single PTV was used throughout the treatment course without any reduction. The total dose was planned in order to achieve the $90 \%$ isodose curve covering $100 \%$ PTV. A daily dose of 2.0 Gy with 5 fractions per week was administered until the total prescribed dose had been delivered. Patients were assessed for toxicities on a weekly basis during 3-DCRT.

\section{Follow-up analysis}

All patients were followed up at a 4-6-week interval until death or their last follow-up (before June 30, 2015). The response of HCC and stent patency was evaluated by abdominal contrast enhanced CT scan (Figure 3B). According to the modified response evaluation criteria in solid tumor (mRECIST) as recommended by American Association for the Study of Liver Diseases [34], the response was classified as complete response (CR), partial response (PR), stable disease (SD) and/or progressive disease (PD). Objective response was defined as the sum of CR and PR. Laboratory tests were performed to evaluate liver and renal function, blood cell count, and coagulation parameters.

Repeated TACE was performed either on detecting enhancement of the residual tumor on the arterial phase, occurrence of a new lesion or both. Indirect portography was undertaken to evaluate the patency of the stent during repeated TACE. TACE was suspended if there was decompensation of liver function or decline in clinical status.

\section{Efficacy and safety assessment}

A low-attenuation intraluminal filling defect that extended from the intrahepatic portal vein branches adjacent to the primary tumor into the MPV was defined as portal vein tumor thrombosis (PVTT).

Overall survival, progression free survival, stent patency period and treatment-related adverse events were compared between the two groups. Survival time was defined as the period from the day of stent placement to patients' death or to their last follow-up. Occurrence of intrahepatic/extrahepatic HCC spread, variceal bleeding and liver function decompensation (e.g., uncontrolled ascites or hepatic encephalopathy) were considered as disease progression. Therefore, progression free survival was the time from first therapy to the presence of one or more of above mentioned events or to the patient's death. Stent occlusion was defined as no contrast medium visualized inside the stent on the portal phase of the contrast enhanced CT scan or indirect portography during the repeat TACE procedures. The stent patency period was calculated as the interval of the day of stent placement and stent occlusion or the day of last follow-up. Treatment-related adverse events were scored according to the Common Terminology Criteria for Adverse Events, version 3.0 [35].

\section{Statistical analysis}

Continuous variables were presented as mean values \pm standard deviation and compared by the independent or paired sample $t$ test. Categorical variables were presented as frequencies and compared using the chi-square test. The overall survival, progression free survival and stent patency period were analyzed with the Kaplan-Meier curves and log-rank test. A $P$ value $<0.05$ was considered statistically significant. Variables that achieved statistical significance $(P<0.05)$ in univariate analysis were subsequently assessed by multivariate analysis by Cox proportional hazards model. A stepwise regression procedure was used to determine the factors that were major independent predictors for survival. SPSS version 19.0 software (SPSS, Chicago, IL) was used for statistical analysis.

\section{ACKNOWLEDGMENTS}

This work was funded by the Advanced and Appropriate Technology Promotion Project from Shanghai Health Family Planning Committee (2013SY060), Scientific Research Program of Shanghai Municipal Heath Bureau (20124188) (Jian-Jun Luo) and National Science Fund for young scholar (81201170) (Ling-Xiao Liu)

\section{CONFLICTS OF INTEREST}

The authors declare no conflicts of interest.

\section{Author contribution}

Study concept and design by JJL and ZPY; acquisition of data by TZY, WZ, QXL, ZHZ, WHL and $\mathrm{JQM}$; analysis and interpretation of data by JHW and BC; obtained funding by JJL and LXL.

\section{REFERENCES}

1. Yang JD, Roberts LR. Hepatocellular carcinoma: A global view. Nat Rev Gastroenterol Hepatol. 2010; 7: 448-58. doi: 10.1038/nrgastro.2010.100 nrgastro.2010.100 [pii].

2. Katagiri S, Yamamoto M. Multidisciplinary treatments for hepatocellular carcinoma with major portal vein tumor thrombus. Surg Today. 2014; 44: 219-26. doi: 10.1007/ s00595-013-0585-6.

3. Minagawa M, Makuuchi M. Treatment of hepatocellular carcinoma accompanied by portal vein tumor thrombus. World J Gastroenterol. 2006; 12: 7561-7. 
4. Bruix J, Sherman M. Management of hepatocellular carcinoma: an update. Hepatology. 2011; 53: 1020-2. doi: 10.1002/hep.24199.

5. Llovet JM, Ricci S, Mazzaferro V, Hilgard P, Gane E, Blanc JF, de Oliveira AC, Santoro A, Raoul JL, Forner A, Schwartz M, Porta C, Zeuzem S, et al. Sorafenib in advanced hepatocellular carcinoma. N Engl J Med. 2008; 359: 378-90. doi: 10.1056/NEJMoa0708857 359/4/378 [pii].

6. Cheng AL, Kang YK, Chen Z, Tsao CJ, Qin S, Kim JS, Luo R, Feng J, Ye S, Yang TS, Xu J, Sun Y, Liang H, et al. Efficacy and safety of sorafenib in patients in the AsiaPacific region with advanced hepatocellular carcinoma: a phase III randomised, double-blind, placebo-controlled trial. Lancet Oncol. 2009; 10: 25-34. doi: 10.1016/S14702045(08)70285-7 S1470-2045(08)70285-7 [pii].

7. Shao YY, Huang CC, Liang PC, Lin ZZ. Hepatic arterial infusion of chemotherapy for advanced hepatocellular carcinoma. Asia Pac J Clin Oncol. 2010; 6: 80-8. doi: 10.1111/j.1743-7563.2010.01287.x AJCO1287 [pii].

8. Lee DS, Seong J. Radiotherapeutic options for hepatocellular carcinoma with portal vein tumor thrombosis. Liver Cancer. 2014; 3: 18-30. doi: 10.1159/000343855 lic0003-0018 [pii].

9. Lau WY, Sangro B, Chen PJ, Cheng SQ, Chow P, Lee RC, Leung T, Han KH, Poon RT. Treatment for hepatocellular carcinoma with portal vein tumor thrombosis: the emerging role for radioembolization using yttrium-90. Oncology. 2013; 84: 311-8. doi: 10.1159/000348325 000348325 [pii].

10. Xue TC, Xie XY, Zhang L, Yin X, Zhang BH, Ren ZG. Transarterial chemoembolization for hepatocellular carcinoma with portal vein tumor thrombus: a metaanalysis. BMC Gastroenterol. 2013; 13: 60. doi: 10.1186/1471-230X-13-60 1471-230X-13-60 [pii].

11. Zhang XB, Wang JH, Yan ZP, Qian S, Liu R. Hepatocellular carcinoma invading the main portal vein: treatment with transcatheter arterial chemoembolization and portal vein stenting. Cardiovasc Intervent Radiol. 2009; 32: 52-61. doi: 10.1007/s00270-008-9454-X.

12. Chung GE, Lee JH, Kim HY, Hwang SY, Kim JS, Chung JW, Yoon JH, Lee HS, Kim YJ. Transarterial chemoembolization can be safely performed in patients with hepatocellular carcinoma invading the main portal vein and may improve the overall survival. Radiology. 2011; 258: 627-34. doi: 10.1148/radiol.10101058 258/2/627 [pii].

13. Zhang W, Luo J, Liu Q, Ma J, Qu X, Yang M, Yan Z, Wang J. Brachytherapy with Iodine- 125 seeds strand for treatment of main portal vein tumor thrombi: an experimental study in a rabbit model. Am J Cancer Res. 2016; 6: 587-99.

14. Luo JJ, Zhang ZH, Liu QX, Zhang W, Wang JH, Yan ZP. Endovascular brachytherapy combined with stent placement and TACE for treatment of HCC with main portal vein tumor thrombus. Hepatol Int. 2016; 10: 185-95. doi: 10.1007/s12072-015-9663-8 10.1007/s12072-015-9663-8 [pii].
15. Luo J, Yan Z, Liu Q, Qu X, Wang J. Endovascular placement of iodine-125 seed strand and stent combined with chemoembolization for treatment of hepatocellular carcinoma with tumor thrombus in main portal vein. $\mathrm{J}$ Vasc Interv Radiol. 2011; 22: 479-89. doi: 10.1016/j. jvir.2010.11.029 S1051-0443(10)01149-8 [pii].

16. Zhang XB, Wang JH, Yan ZP, Qian S, Du SS, Zeng ZC. Hepatocellular carcinoma with main portal vein tumor thrombus: treatment with 3-dimensional conformal radiotherapy after portal vein stenting and transarterial chemoembolization. Cancer. 2009; 115: 1245-52. doi: 10.1002/cncr.24139.

17. Nath R, Anderson LL, Luxton G, Weaver KA, Williamson JF, Meigooni AS. Dosimetry of interstitial brachytherapy sources: recommendations of the AAPM Radiation Therapy Committee Task Group No. 43. American Association of Physicists in Medicine. Med Phys. 1995; 22: 209-34.

18. Zhang W, Yan Z, Luo J, Fang Z, Wu L, Liu Q, Qu X, Liu L, Wang J. Iodine-125 seeds strand for treatment of tumor thrombus in inferior vena cava: an experimental study in a rabbit model. Cardiovasc Intervent Radiol. 2013; 36: 137182. doi: 10.1007/s00270-013-0628-9.

19. Chen Y, Wang XL, Yan ZP, Wang JH, Cheng JM, Gong GQ, Luo JJ. The use of (1)(2)(5)I seed strands for intraluminal brachytherapy of malignant obstructive jaundice. Cancer Biother Radiopharm. 2012; 27: 317-23. doi: 10.1089/ cbr.2011.0999.

20. Liu L, Zhang C, Zhao Y, Qi X, Chen H, Bai W, He C, Guo W, Yin Z, Fan D, Han G. Transarterial chemoembolization for the treatment of advanced hepatocellular carcinoma with portal vein tumor thrombosis: prognostic factors in a singlecenter study of 188 patients. Biomed Res Int. 2014; 2014: 194278. doi: 10.1155/2014/194278.

21. Jeong SW, Jang JY, Shim KY, Lee SH, Kim SG, Cha SW, Kim YS, Cho YD, Kim HS, Kim BS, Kim KH, Kim JH. Practical effect of sorafenib monotherapy on advanced hepatocellular carcinoma and portal vein tumor thrombosis. Gut Liver. 2013; 7: 696-703. doi: 10.5009/gnl.2013.7.6.696.

22. Zhu K, Chen J, Lai L, Meng X, Zhou B, Huang W, Cai M, Shan H. Hepatocellular carcinoma with portal vein tumor thrombus: treatment with transarterial chemoembolization combined with sorafenib--a retrospective controlled study. Radiology. 2014; 272: 284-93. doi: 10.1148/ radiol.14131946.

23. Hu H, Duan Z, Long X, Hertzanu Y, Shi H, Liu S, Yang Z. Sorafenib combined with transarterial chemoembolization versus transarterial chemoembolization alone for advancedstage hepatocellular carcinoma: a propensity score matching study. PLoS One. 2014; 9: e96620. doi: 10.1371/journal. pone.0096620 PONE-D-13-54600 [pii].

24. Zhang Y, Fan W, Wang Y, Lu L, Fu S, Yang J, Huang Y, Yao W, Li J. Sorafenib With and Without Transarterial Chemoembolization for Advanced Hepatocellular Carcinoma With Main Portal Vein Tumor Thrombosis: A Retrospective Analysis. Oncologist. 2015; 20: 1417-24. doi: 
10.1634/theoncologist.2015-0196 theoncologist.2015-0196 [pii].

25. Zhang JQ, Huang XQ, Zhang J, Cai P, Chen W, Wang J, Zhou DQ, Zhang EQ. [CT guided radioactive seed (125) I implantation in treating multiple bone metastasis]. Zhonghua Yi Xue Za Zhi. 2008; 88: 2739-42.

26. Waksman R, Robinson KA, Crocker IR, Gravanis MB, Cipolla GD, King SB, 3rd. Endovascular low-dose irradiation inhibits neointima formation after coronary artery balloon injury in swine. A possible role for radiation therapy in restenosis prevention. Circulation. 1995; 91: 1533-9.

27. Dawson LA, Ten Haken RK, Lawrence TS. Partial irradiation of the liver. Semin Radiat Oncol. 2001; 11: 2406. doi: S1053429601000340 [pii].

28. Ling CC. Permanent implants using Au-198, Pd-103 and I-125: radiobiological considerations based on the linear quadratic model. Int J Radiat Oncol Biol Phys. 1992; 23: 81-7. doi: 0360-3016(92)90546-T [pii].

29. Yang M, Fang Z, Yan Z, Luo J, Liu L, Zhang W, Wu L, Ma J, Yang Q, Liu Q. Transarterial chemoembolisation (TACE) combined with endovascular implantation of an iodine-125 seed strand for the treatment of hepatocellular carcinoma with portal vein tumour thrombosis versus TACE alone: a two-arm, randomised clinical trial. J Cancer Res Clin Oncol. 2014; 140: 211-9. doi: 10.1007/s00432-013-1568-0.
30. Sindelar WF, Kinsella TJ. Normal tissue tolerance to intraoperative radiotherapy. Surg Oncol Clin N Am. 2003; 12: $925-42$.

31. Liu Y, Liu R, Wang P, Li S, Shen H. Percutaneous implantation of (125)iodine seeds for treatment of portal vein tumor thrombosis in hepatocellular carcinoma. Med Oncol. 2015; 32: 214. doi: 10.1007/s12032-015-0657-0.

32. Bruix J, Sherman M. Management of hepatocellular carcinoma. Hepatology. 2005; 42: 1208-36. doi: 10.1002/ hep. 20933.

33. Shuqun C, Minshan C, Jianqiang C, The National Research Cooperative Group For D, Treatment Of Hepatocellular Carcinoma With Tumor T. Chinese expert consensus on multidisciplinary diagnosis and treatment of hepatocellular carcinoma with portal vein tumor thrombus: 2016 edition. Oncotarget. 2017; 8:8867-8876. doi: 10.18632/ oncotarget.12817.

34. Lencioni R, Llovet JM. Modified RECIST (mRECIST) assessment for hepatocellular carcinoma. Semin Liver Dis. 2010; 30: 52-60. doi: 10.1055/s-0030-1247132.

35. National Cancer Institute. Common terminology criteria for adverse events, version 3.0. http://ctep.cancer.gov/reporting/ ctc. html. Published 9 August 2006. Accessed 18 June 2009. 